\title{
Evolutive optimisation in biological wastewater treatment
}

\author{
Prof. Dr. Franz Xaver Wildenauer, Prof. Dr. habil. Rolf Hirte, Dipl.-Ing. (FH) Stephan Zillekens
}

\begin{abstract}
A number of industrial waste waters show a non balanced compostion of inorganic nutrients, caused in the specific production process. In independence of the waste water treatment plant, the biodegradability rate of these sewage is often limited by several parameters. The consequences are a high susceptibility to trouble and problems to observe the limits of COD and other parameters. Subsequently a modified method of evolutive strategies is described as an alternative to the classical methods to eliminate limiting factors. The results are based on industrial waste water containing high amounts of surfactants (12.000 mg/l COD)
\end{abstract}

\section{Introduction}

In comparison to microbial media, at disposal industrial wastewater has neither a balanced proportion of carbon, nitrogen and phosphorus, nor a sufficient supply of other salts and trace elements. In consequence, the variety and activity of the microbial population is reduced. Examples are sabage from plastic-production, detergent-production and the metal manufacturing industry. Even wastewater found in food-production plants often does not contain the necessary nutrients concerning biological demand. It is known, that by altering the amount of nutrients in, anaerobic and aerobic wastewater plants, the velocity of biodegradation can be improved. This corresponds to an increase of plant capacity without additional investment costs. In addition to an increase of sludge sedimentation and a greater safety in operation, higher elemination rates for phosphorus and nitrogen can be detected.

At the moment, putting additives to wastewater is common practise without any knowledge of the exact chemical compostion of the sewage and the specific nutrient demand of the micro-organisms, thus most improvements are achieved by chance. Up to now two methods have been used to increase the biodegradation of wastewater, as the costs for systematic investigations are too high:

Firstly the „blind" dosage of the most important nutrients phosphorus and nitrogen. In this case, the $\mathrm{C}-\mathrm{N}$ $\mathrm{P}$ proportion is detected in a sample of activated sludge from the plant, and the missing nutrients are added according to information from the literature. The nonspecific character of this method is obvious, as literature data concerning the nutrient proportion varies between very different levels (Fiechter et al, 1984), and only phosphorus and nitrogen are taken into consideration. Secondly, a nutrient composition can be determined by optimisation of one additive after the other. Usually this classical optimisation is to expensive for the "waste“ water and mutual influences of the additives are disregarded.
Based on these facts, a study at the TFH Wildau was initiated to find out whether the mathematical method called „evolutive optimisation“ could be used to increase the biodegradability of industrial wastewater. Additionally a standard procedure for this optimisation should be developed and the optimisation -software, up to now used only in computer simulation-models, should be adapted to the requirements for practise use.

\section{Methods}

\section{Evolutive Strategies}

„Evolutive optimizations“ are mathematical methods, developed already in the sixties (Goldberg et al. Rechenberger et al 1973), for the optimisation of complex systems influenced by a number of different parameters. Computer programs based on these methods simulate the natural process of the evaluative adaptation and optimisation by mutation and selection to achieve the defined aim of optimisation.

Repeatedly variation of parameters and following valuation of the achieved effects on the system being examined, lead to a successive approach to an optimum. As a matter of principle, this method can be used for very different optimisation problems, started with complicated static constructions up to design of the optimal aminoacid sequences for polypeptides with defined features.

In all cases, the optimisation has to start with the choice of the parameters that should be varied and examined to determine their influence on the system. After that, minimal and maximal limiting values for the chosen parameters and intervals between these limits must be determined. This data is being digitized and, analogue biological genes in DNA, arranged in linear sequences. The most important step is defining the optimum afterwards. After these preparations, the quality of several parameter combinations can be tested and, as a result, quality factors can be appointed to each combination. According to the number of combinations (,individuals"), which can be tested at the same time, the parameter combinations for the first "generation“ are chosen by the program as a fortuitous result. After testing this first generation, the detected quality factors of each individual are the basic elements for calculating the next generation. On this calculation, partly the best individuals of the first generation are taken into consideration. Simultaneous, new individuals are being created from two data sequences by mutations like crossover, deletions and insertions. The calculated second generation, based on „experiences“ acqired in the first generation, is tested once more. By permanent repe- 
tition of this procedure, the quality of the individuals approaches to the defined optimum after several generations. The optimisation is stopped when the increase of quality in two following generations is nearly zero. The result of the optimisation is the parameter combination of the best individual from the last generation. The advantage of this optimisation compared to other methods is the low number of tests necessary for enormous increase of quality. For example, the optimum of a system, influenced by 5 parameters varied in 10 intervals, is one of 105 possible combinations. Considering our experience, good results can be achieved with about 80 tests, assuming 10 individuals in each generation. As a disadvantage, the results do not always correspond to the absolute optima. Concerning the relation between costs and increase in complicated systems, older methods can't compare with evolutive optimisations.

Evolutive optimisation for the system wastewater/ activated sludge

For our experiment the method of evolutive optimisation had to be adapted to the system industrial wastewater in combination with activated sludge. The sewage we choose was taken from a detergent-producing plant, having a COD of about $12.000 \mathrm{mg} / \mathrm{l}$ that was mainly caused by anionic and non-ionic surfactants. The definition for the optima included two main objectives:

a) The increase of the biodegradation rate should be maximum

b) The sum of additives for this increase should be minimum

To add variables, concentrated solutions of phosphorus, nitrogen, sulphate, magnesium, iron, molasses and a mixture of trace elements were added to the water. The biodegradation rate in batch cultures was measured respiratory. The oxygen demand of a stirred and temperature controlled wastewater sample can be detected and compensated by defined quantities of electrolytically produced oxygen (Fig. 1).

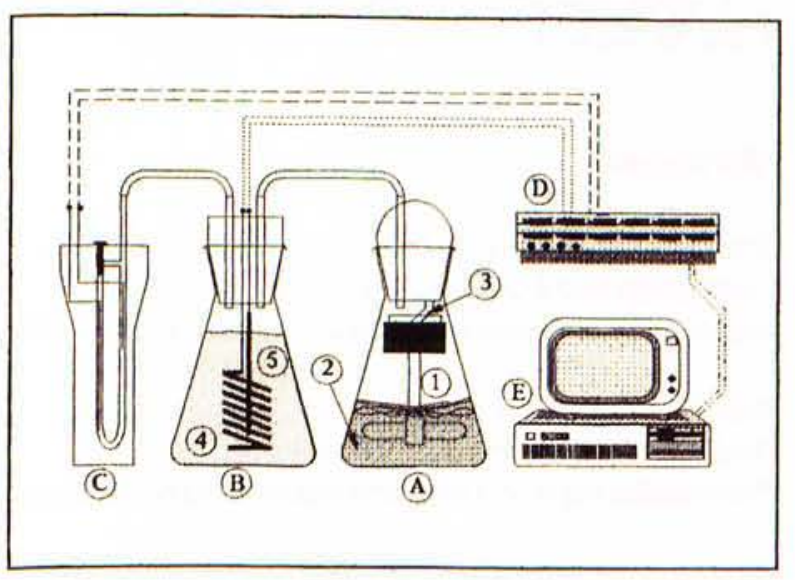

Fig. 1: Respiromatic measurement of oxygen demand

A: Sample, I stirrer,

2 wastewater,

3 CO2-adsorbent

B: Electrolytic oxygen production

C: Manometer

D: Recording instrument

E: Computer
In this arrangement, 10 individuals (samples) could be tested at the same time.

By variation and standardisation of this method, especially using inocula with equal activities and modifying the mathematical software, the reproducibility of the measurements allowed the application of evolutive optimisation strategies (Fig. 2).

\begin{tabular}{|c|}
\hline Choice of wastewater \\
\hline$\Downarrow$ \\
\hline Definition aim of optimisation \\
\hline$\Downarrow$ \\
\hline choice of additives \\
\hline$\Downarrow$ \\
\hline Preliminary tests \\
\hline$\Downarrow$ \\
\hline $\begin{array}{l}\text { Parameter combination for the } 1 \text {. generation } \\
\text { as a fortuitous result }\end{array}$ \\
\hline$\Downarrow$ \\
\hline Degradation test 1. (n.) generation \\
\hline$\Downarrow$ \\
\hline Valuation of the results, \\
\hline$\Downarrow$ \\
\hline Input of the detected quality factors \\
\hline$\Downarrow$ \\
\hline $\begin{array}{l}\text { Calculation of the parameter combinations for } \\
\text { the } 2 \text {. } \\
\qquad(n+1) \text { generation, etc. }\end{array}$ \\
\hline$\Downarrow$ \\
\hline Aim of optimisation \\
\hline
\end{tabular}

Fig. 2: Procedure to determine the optimal nutrient composition using „evolutive optimisations"

In preliminary tests the general effect of the additives was determined by addition in concentration excess. These tests contained $50 \%$ of the maximal possible limit values of each additive defined before. The measured degradation rate was compared with a sample without any additives (blank test). The result of the optimisation, achieved in batch cultures, was afterwards verified in continous culture, to test the biodegradability under conditions that occurs in wastewater treatment plants.

\section{Results}

The result of the preliminary test after 180 hour's experimentation time is shown in fig. 3a. As the additives were added in concentration excess (compare to the demand after optimisation) the flattening of the oxygen demand curve after 50 hours experimental time is remarkable. This effect, supposedly caused by diauxie similar - phenomenon, is a sign for the additives-concentrations. The unspecific addition of salts caused an increase of the biodegradation rate of about $600 \%$. compared with the sample containing no additives.

Considering this result, the optimisation was started. The development of the additive amounts necessary for equal or enhanced biodegradation rates within 9 gene- 


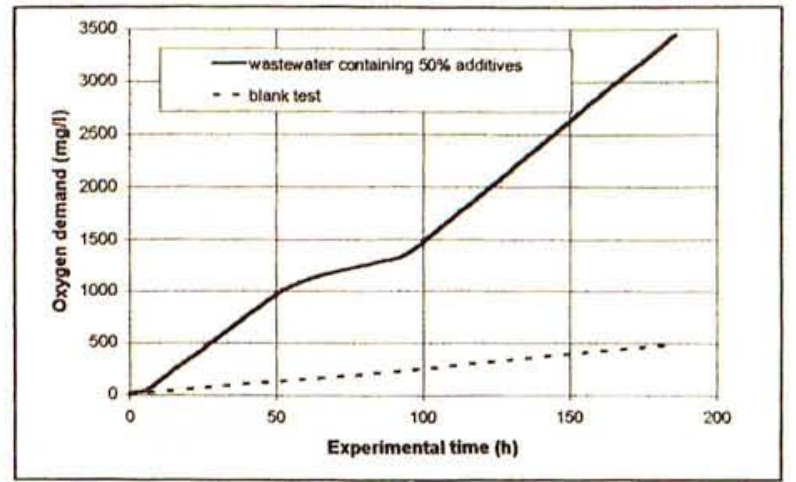

Fig. 3a: Result of the preliminary test: Added amount of additives: $50 \%$

Oxygen demand without additives: $490 \mathrm{mg} / \mathrm{l}$

Oxygen demand after unspecific addition $3450 \mathrm{mg} / \mathrm{l}$

rations is shown in fig. 4 . In this figure, the sum of additives is given in percentage of the maximum possible amount. To make the general development of the necessary additive amounts clear, the graphic contains the amounts needed for a whole generation and for the best individual of each generation. It is obvious, that both, the generations and the best individuals, contain fewer additives from generation to generation.

The „compostion“ of additives detected after 9 generations as the result of optimisation was added to a waste water sample and compared with a blank test again (see fig. 3b).

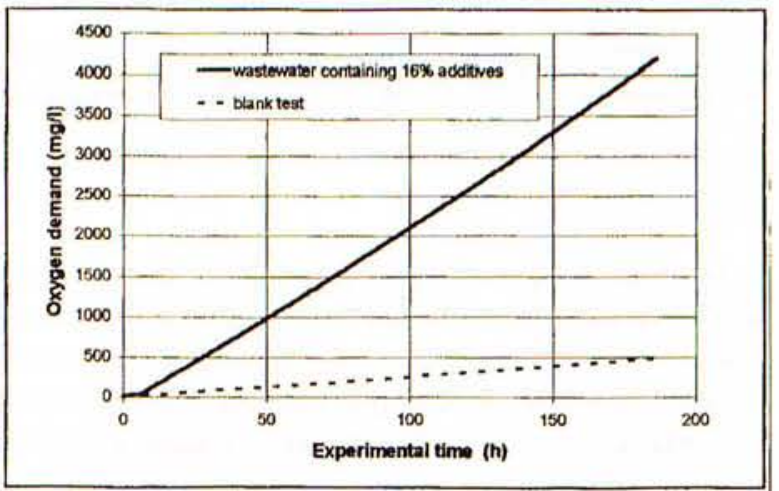

Fig. 3b: Gained optimisation results:

Added amount of additives: $16 \%$

Oxygen demand without additives: $490 \mathrm{mg} / \mathrm{l}$

Oxygen demand after optimisation: $4190 \mathrm{mg} / \mathrm{l}$

The biodegradation rate increased to $755 \%$ with an additive demand of about $16 \%$ of the maximal possible amount. In comparison to the preliminary test, the biodegradation rate enhanced up to $25 \%$, containing only $30 \%$ of the additives added in the preliminary test. In the following tests in continuous culture, the biodegradation rate from $200 \mathrm{mg} 1-1 \mathrm{~d}-1$ COD without additives increased up to $390 \mathrm{mg} 1-1 \mathrm{~d}-1$ COD containing the additives in concentrations investigated by evolutive optimisation.

\section{Conclusions}

In the described examinations, the optimisation strategy was mathematical modified and a standard proce-

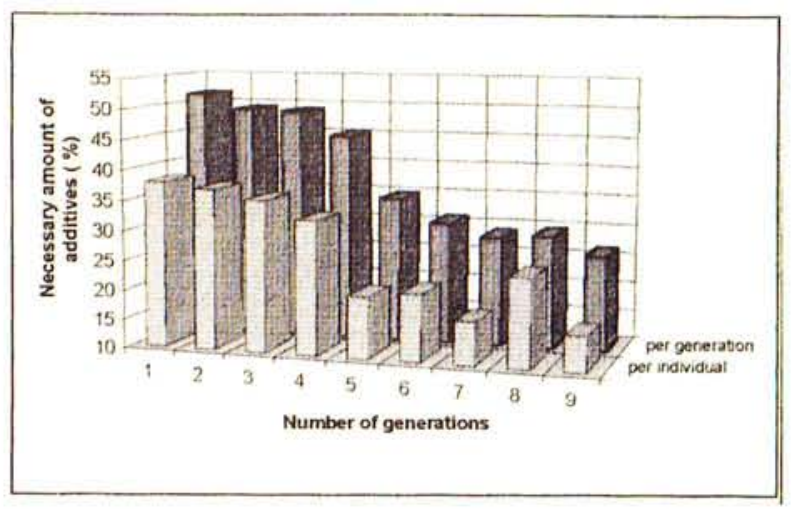

Fig 4: This figure shows the development of necessary amounts of additives to gain equal or enhanced biodegradation rates. The sum of additives is given in percentage of the maximum possible amount for a whole generation and for the best individum of each generation

dure for using these strategies for industrial wastewater was developed. We have shown successfully, that with a minimal insert of additives high increases of biodegradation can be gained. The increase of the biodegradation rates by adding limiting additives enhances the capacity of existing wastewater treatment plants. Especially plants running at maximal capacity can avoid breaking limiting values by using this optimisation method. The most important advantage is, that additional investment costs are not necessary. The disadvantage is, that the wastewater should show a nearly constant composition. However, this requirement is met by most of the industrial wastewater found in industrial production.

The application of this optimisation method is not reduced to wastewater treatment at all. It generally can be used for the supply of the specific nutrient demand of microorganismns. Examples are the treatment of exhausted air in biofilters in the biological treatment of contaminated soil.

\section{Acknowledgements}

The authors express their appreciation to the ministry of science, research and culture, Brandenburg, for the financial support.

\section{Verfasser}

Prof. Dr. F.ranz Xaver Wildenauer Technische Fachhochschule Wildau Fachbereich Ingenieurwesen/Wirtschaftsingenieurwesen

Prof. Dr. habil. Rolf Hirte

Technische Fachhochschule Wildau

Fachbereich Ingenieurwesen/Wirtschaftsingenieurwesen

Dipl.-Ing. (FH) Stephan Zillekens Technische Fachhochschule Wildau Fachbereich Ingenieurwesen/Wirtschaftsingenieurwesen Labor für Bioverfahrenstechnik 